\title{
APPLING THE FORWARD KINEMATIC OF THE BIPED ROBOT WITH 12 DOF BASED DENAVIT AND HARTENBERG METHOD
}

\begin{abstract}
This paper presents Forward kinematics analysis for the Biped Robot Model (AK robot) and focuses on the considered lower body of a 12 DOF biped robot. Each leg modeled as a kinematic chain with Seven links connected by Six revolute joints. The Description and parameters of AK robot was introduced, also in this paper studied the forward kinematics that refers the analytical study. For describing robot kinematics was used Denavit \& Hartenberg (D-H) [1] method, which shows that a general transformation between two joints requires four parameters. These parameters known as the D-H parameters of D-H notation which allows the step from a link to the following link by 4 basic transformations that depends only on the robot's constructive characteristics
\end{abstract}

Keywords: Biped Robot, Denavit \& Hartenberg, Forward Kinematic.

\section{INTRODUCTION}

Humanoid robotics is one of the most exciting fields of research in the present robotic research area. AK robot kinematics pertains to the motion of bodies in a robotic mechanism without regard to the forces/torques that cause the motion. Since robotic mechanisms are by their very essence designed for motion, kinematics is the most fundamental aspect of robot design, analysis, control and simulation [2].

The kinematic model of an AK Biped Robot can be represented by the homogeneous transformation matrix. These are basic transformations that relate the reference system of the element $n+1$ with the reference system of the element $n$ [3].

\section{AK BIPED ROBOT MODEL}

The 12-DOF of AK Robot is (see Figure 1) shown in the initial state with all zero joint angles, the 12-DOF summarized in Table 1. Note that last hip joint, the knee joint, and the first ankle joint are all about parallel $\mathrm{Z}$ axes.

Table 1. Degrees of freedom of lower body biped robot

\begin{tabular}{|l|l|l|l|}
\hline & \multicolumn{3}{|c|}{ Degree of freedom } \\
\hline & Right leg & Left Leg & Two legs \\
\hline Hip (roll/pitch/yaw) & 3 DOF & 3 DOF & 6 DOF \\
\hline Knee (pitch) & 1 DOF & 1 DOF & 2 DOF \\
\hline Ankle (roll/pitch) & 2 DOF & 2 DOF & 4 DOF \\
\hline & \multicolumn{4}{r}{ Total } & 12 DOF \\
\hline
\end{tabular}

\section{AK PARAMETERS}

The length parameters of AK robot are given first, then the joint angle limitation, and at the last the D-H parameters for the AK Biped Robot Model presented.

The parameters values of torso reference frame $\mathrm{L}_{\mathrm{TX}}, \mathrm{L}_{\mathrm{TY}}, \mathrm{L}_{\mathrm{TZ}}$ introduces in Table 2, these parameters doesn't considers as D-H Parameters and will be used in forward kinematics solutions, where $\mathrm{L}_{1}, \mathrm{~L}_{2}, \mathrm{~L}_{\mathrm{F}}$ will use as a D-H paraments. 


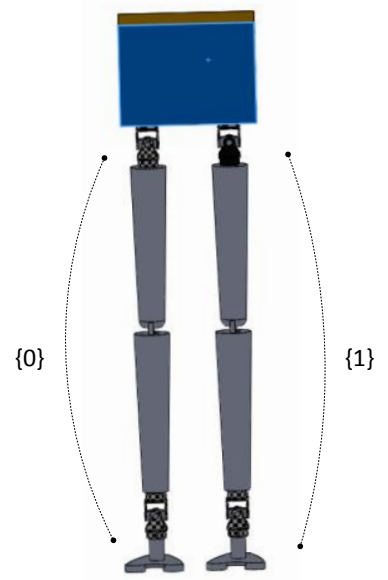

Figure 1. AK Biped Robot Model robot, Zero State

Table 2. AK Torso Dimensions

\begin{tabular}{|c|r|c|}
\hline \multirow{2}{*}{ Links Description } & \multicolumn{2}{|c|}{ Dimensions } \\
\cline { 2 - 3 } & Symbol & Unit $(\mathrm{mm})$ \\
\hline Torso to leg on X axis & $\mathrm{L}_{\mathrm{TX}}$ & 15 \\
\hline Torso to leg on Y axis & $\mathrm{L}_{\mathrm{TY}}$ & 150 \\
\hline Torso to leg on Z axis & $\mathrm{L}_{\mathrm{TZ}}$ & 35 \\
\hline Length hip to knee & $\mathrm{L}_{1}$ & 230 \\
\hline Length knee to foot & $\mathrm{L}_{2}$ & 225 \\
\hline High of foot & $\mathrm{L}_{\mathrm{F}}$ & 47 \\
\hline
\end{tabular}

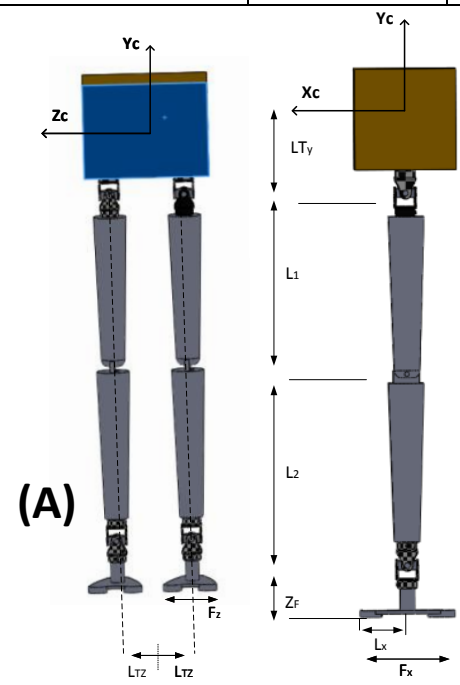

(B)

Figure 2. AK Biped Robot Lengths Definition (A) front side, (B)Leg

The AK foot (see Figure 2) dimensions valuse summurized shown on Table 3.

Table 3 . AK Foot Dimensions

\begin{tabular}{|l|c|l|}
\hline \multirow{2}{*}{ Links Description } & \multicolumn{2}{|c|}{ Dimensions } \\
\cline { 2 - 3 } & $\begin{array}{c}\text { Sy } \\
\text { mbol }\end{array}$ & Unit $(\mathrm{mm})$ \\
\hline Length Foot on X axis & $\mathrm{F}_{\mathrm{x}}$ & 104.00 \\
\hline Length Foot on Y axis & $\mathrm{F}_{\mathrm{y}}$ & 15.00 (10.0 front) \\
\hline Length Foot on Z axis & $\mathrm{F}_{\mathrm{z}}$ & 42 \\
\hline $\mathrm{Y}_{\mathrm{c}}$ to front of foot & $\mathrm{L}_{\mathrm{x}}$ & 52.00 \\
\hline
\end{tabular}




\begin{tabular}{|l|l|l|}
\hline Between foot & $\mathrm{L}_{\mathrm{z}}$ & 23.00 \\
\hline
\end{tabular}

The kinematic joint angle limits for the Biped Robot are specific hardware design values associated with the D-H Parameters given in Table 4. The synthesis of the kinematic chains is based on human body parameters in terms of ratios, range of motion, and physical length [4]. Table 4 shows the range of motion for the human leg, while the parameters corresponding to the robot leg are based on a data in [2].

Table 4. AK Joint range of motion

\begin{tabular}{|c|c|c|c|c|}
\hline \multicolumn{3}{|c|}{ Joint } & \multirow{2}{*}{ Axis } & \multirow{2}{*}{$\begin{array}{l}\text { Humanoid } \\
\text { robot (deg.) }\end{array}$} \\
\hline Joint $i$ & \multicolumn{2}{|c|}{ Joint Name } & & \\
\hline \multicolumn{5}{|c|}{ Right Le } \\
\hline 7 & \multirow{3}{*}{ Hip } & Yaw & $Z_{1}$ & -150 to 45 \\
\hline 11 & & Roll & $Z_{2}$ & 0 to 60 \\
\hline 9 & & Pitch & $Z_{3}$ & -100 to -30 \\
\hline 13 & Knee & Yaw & $Z_{4}$ & 0 to 130 \\
\hline 17 & \multirow{2}{*}{ Ankle } & Pitch & $Z_{5}$ & -60 to 60 \\
\hline 15 & & Roll & $Z_{6}$ & -30 to 60 \\
\hline \multicolumn{5}{|c|}{ Left Leg } \\
\hline 8 & \multirow{3}{*}{ Hip } & Yaw & $Z_{1}$ & -45 to 150 \\
\hline 12 & & Roll & $Z_{2}$ & 60 to 0 \\
\hline 10 & & Pitch & $Z_{3}$ & -30 to 100 \\
\hline 14 & Knee & Yaw & $Z_{4}$ & -130 to 0 \\
\hline 18 & \multirow{2}{*}{ Ankle } & Pitch & $Z_{5}$ & -60 to 60 \\
\hline 16 & & Roll & $Z_{6}$ & -30 to 60 \\
\hline
\end{tabular}

Note that some ranges of motion of the humanoid robot do not correspond to the human leg, due to the interference between mechanical parts.

The standardized Denavit-Hartenberg (D-H) Parameters [5] are used to describe the links/joints geometry of a serial- chain robot. Next the D-H Parameters are presented for the Biped Robot serial chain, i.e. right legs.

The Cartesian reference frame definitions for two AK Biped Robot serial chain (6-dof right leg) are given in Figure 2 Ошибка! Источник ссылки не найден.. It is important to note that all of these D-H parameters shown in the zero-angle poses.

Therefore, it looks like many moving coordinate frame axes line up, but this will change as joint angles rotate away from zero in all cases. 

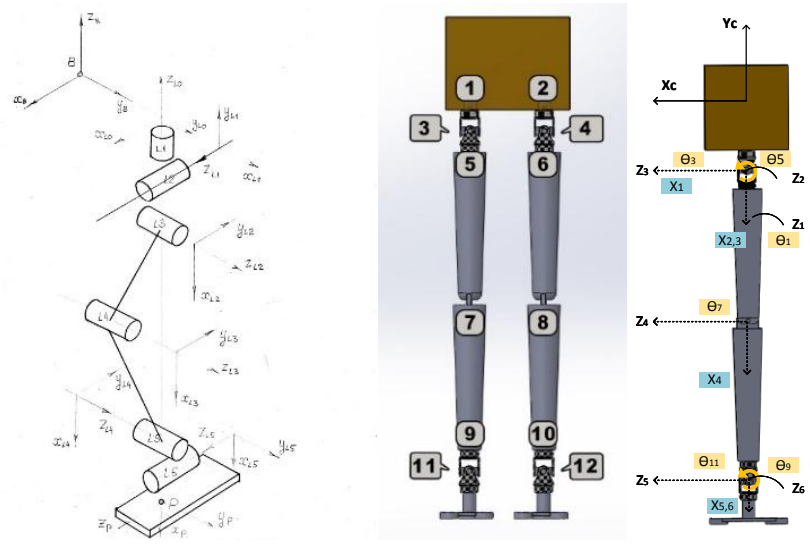

Figure 3. Left: AK Biped Robot Angles Numbering Right: Six-DOF Right Leg Coordinate Frames

We present D-H parameters for the 6-dof right leg (the 6-dof left leg are symmetric to right leg, as seen in Figure 3. gives the D-H parameters for the 6-DOF right leg.

Table 5. AK Biped Robot Right Leg D-H parameters

\begin{tabular}{|c|c|c|c|c|c|c|}
\hline $\mathrm{i}$ & Link & joint & $a_{i-1}[\mathrm{~mm}]$ & $\alpha_{i-1}\left[^{\circ}\right]$ & $d_{i}[\mathrm{~mm}]$ & $\theta_{i}\left[^{\circ}\right]$ \\
\hline 1 & Waist & None & 0 & 0 & 0 & $\theta_{7}$ \\
\hline 2 & $90^{\circ}$ Joint Top & Waist-Lateral & 90 & 0 & 0 & $\theta_{5}+90$ \\
\hline 3 & Thigh & Waist - Thigh & 90 & 0 & 0 & $\theta_{3}$ \\
\hline 4 & Shank & Knee & 0 & $L_{1}$ & 0 & $\theta_{7}$ \\
\hline 5 & $90^{\circ}$ Joint Down & Ankle- Lateral & 0 & $L_{2}$ & 0 & $\theta_{11}$ \\
\hline 6 & Foot & Ankle & -90 & 0 & 0 & $\theta_{9}$ \\
\hline
\end{tabular}

\section{AK KINEMATIC MODELING}

In general, the kinematics of biped robot describes the motion of the point or body joints without considering the motion. Given the joint values, we calculate the pose (position and orientation) of the end frame of interest where the end frame (end-effector) is the robot foot.

In this paper, the kinematic modeling of the AK robot derived by using the DenavitHartenberg (D-H) notation.

\section{Forward Kinematics}

Denavit-Hardenberg formulation is used to model AK robot. Each part is considered as a link represented by a line along its joint axis and common normal to next joint axis.

It depends on substituting each line of the D-H parameters table into transformation matrix. giving the position of frame number $\mathrm{i}$ with respect to its back along frame i-1.

As we can note that both legs are identical and $\theta_{i}$ is the only parameter that is changing (angle of each leg's joint). Together, these four transformations in (1) lead to a unique homogeneous transformation matrix with four variables representing the relationship between these two links. The transformation matrices are calculated using the following general form of transformation matrix.

$$
{ }_{i}^{i-1} T=\left[\begin{array}{cccc}
s \theta_{i} & c \theta_{i} & 0 & a_{i-1} \\
s \theta_{i} c \alpha_{i-1} & c \theta_{i} c \alpha_{i-1} & -s \alpha_{i-1} & -d_{i} s \alpha_{i-1} \\
s \theta_{i} s \alpha_{i-1} & c \theta_{i} c \alpha_{i-1} & c \alpha_{i-1} & d_{i} c \alpha_{i-1} \\
0 & 0 & 0 & 1
\end{array}\right]
$$

Where $s \theta_{i}=\sin \theta_{i}, C \theta_{i}=\cos \theta_{i}, d_{i}-1, a_{i}-1$, and $\alpha_{i}+1$ are the D-H parameters for the $i$ link. Hence, is sufficient to distinguish the $\theta_{i}-1, d_{i}-1, a_{i}-1, \alpha_{i}-1$ parameters to obtain the $\left[{ }^{i-1} T\right]$ matrices and relate each AK robot's link. 


\section{A. AK Right Leg Forward Kinematic}

The problem of forward kinematic of right leg serial chain which has 6 DOF for giving the joints angles values $\left(\theta_{1}, \theta_{5}, \theta_{3}, \theta_{7}, \theta_{11}, \theta_{9}\right)$ is to calculate the $\left[{ }_{6}^{0} T\right]$ and $\left[{ }_{F}^{C} T\right]$, where the $\mathrm{F}$ is the endeffector of leg which is the right foot and $\mathrm{C}$ is the AK robot chest reference frame. By Substituting each row of

the

D-H parameters in Table 5. into the matrix for $\left[{ }_{i}^{i-1} T\right]$ will get a six neighboring homogeneous transformation matrices as a function of the joint angles like following.

$$
\begin{gathered}
{\left[{ }_{1}^{0} T\right]=\left[\begin{array}{cccc}
C_{1} & -S_{1} & 0 & 0 \\
S_{1} & C_{1} & 0 & 0 \\
0 & 0 & 1 & 0 \\
0 & 0 & 0 & 1
\end{array}\right] \quad\left[{ }_{2}^{1} T\right]=\left[\begin{array}{cccc}
-S_{5} & -C_{5} & 0 & 0 \\
0 & 0 & -1 & 0 \\
-C_{5} & -S_{5} & 0 & 0 \\
0 & 0 & 0 & 1
\end{array}\right]} \\
{\left[{ }_{3}^{2} T\right]=\left[\begin{array}{cccc}
C_{3} & -S_{3} & 0 & 0 \\
0 & 0 & -1 & 0 \\
S_{3} & C_{3} & 0 & 0 \\
0 & 0 & 0 & 1
\end{array}\right]} \\
{\left[{ }_{4}^{3} T\right]=\left[\begin{array}{cccc}
C_{7} & -S_{7} & 0 & L_{1} \\
S_{7} & C_{7} & 0 & 0 \\
0 & 0 & 1 & 0 \\
0 & 0 & 0 & 1
\end{array}\right] \quad\left[{ }_{5}^{4} T\right]=\left[\begin{array}{ccccc}
C_{11} & -S_{11} & 0 & L_{2} \\
S_{11} & C_{11} & 0 & 0 \\
0 & 0 & 1 & 0 \\
0 & 0 & 0 & 1
\end{array}\right]} \\
{\left[{ }_{6}^{5} T\right]=\left[\begin{array}{cccc}
C_{9} & -S_{9} & 0 & 0 \\
0 & 0 & 1 & 0 \\
-S_{9} & -C_{9} & 0 & 0 \\
0 & 0 & 0 & 1
\end{array}\right]}
\end{gathered}
$$

Where the taking after shortened forms were utilized: $C_{i}=\cos \theta_{i}, S_{i}=\sin \theta_{i}$,

for $i=1,5,3,7,11,9$. Now substitute these three neighboring homogeneous transformation matrices into the following homogeneous transform equation to derive the Forward Kinematic result.

$$
\left[{ }_{6}^{0} T\right]=\left[{ }_{1}^{0} T\left(\theta_{1}\right)\right]\left[{ }_{2}^{1} T\left(\theta_{5}\right)\right]\left[{ }_{3}^{2} T\left(\theta_{3}\right)\right]\left[{ }_{4}^{3} T\left(\theta_{7}\right)\right]\left[{ }_{5}^{4} T\left(\theta_{11}\right)\right]\left[{ }_{6}^{5} T\left(\theta_{9}\right)\right]
$$

Since there are three parallel $\mathrm{Z}$ axes $(3,4,5)$ in the right leg, we can group the above matrix multiplications as follows for a significant simplification.

$$
\left[{ }_{6}^{0} T\right]=\left[{ }_{1}^{0} T\left(\theta_{7}\right)\right]\left[{ }_{2}^{1} T\left(\theta_{11}\right)\right]\left[{ }_{5}^{2} T\left(\theta_{3}, \theta_{7}, \theta_{11}\right)\right]\left[{ }_{6}^{5} T\left(\theta_{9}\right)\right]
$$

Where

$$
\left[{ }_{5}^{2} T\right]=\left[\begin{array}{cccc}
C_{9} & -S_{9} & 0 & 0 \\
0 & 0 & 1 & 0 \\
-S_{9} & -C_{9} & 0 & 0 \\
0 & 0 & 0 & 1
\end{array}\right]
$$

Where the abbreviations were used: $C_{a b}=\cos \left(\theta_{3}+\theta_{7}\right), S_{a b}=\sin \left(\theta_{3}+\theta_{7}\right), S_{a b c}=$ $\sin \left(\theta_{3}+\theta_{7}+\theta_{11}\right), C_{a b c}=\cos \left(\theta_{3}+\theta_{7}+\theta_{11}\right), S_{a b c}=\sin \left(\theta_{3}+\theta_{7}+\theta_{11}\right)$ Were used.

The right leg Forward Kinematic result is: 


$$
\left[{ }_{6}^{0} T\right]=\left[\begin{array}{cccc}
r_{11} & r_{12} & r_{13} & P_{x} \\
r_{21} & r_{22} & r_{23} & P_{y} \\
r_{31} & r_{32} & r_{33} & P_{z} \\
0 & 0 & 0 & 1
\end{array}\right]
$$

Where:

$$
\begin{aligned}
& r_{11}=\left(S_{1} S_{a b c}-C_{1} C_{5} C_{a b c}\right) C_{9}-C_{1} C_{5} C_{9} \\
& r_{21}=\left(-C_{1} S_{a b c}-S_{1} S_{5} C_{a b c}\right) C_{9}-S_{1} C_{5} S_{9} \\
& r_{31}=-S_{5} S_{9}+C_{5} C_{9} C_{a b c} \\
& r_{12}=-\left(S_{1} S_{a b c}-C_{1} S_{5} C_{a b c}\right) S_{9}-C_{1} C_{5} C_{9} \\
& r_{22}=-\left(-C_{1} S_{a b c}-S_{1} S_{5} C_{a b c}\right) S_{9}-S_{1} C_{5} C_{9} \\
& r_{32}=-S_{5} C_{9}-C_{5} C_{9} C_{a b c} \\
& r_{13}=S_{1} C_{a b c}+C_{1} S_{5} S_{a b c} \\
& r_{23}=-C_{1} C_{a b c}+S_{1} S_{5} S_{a b c} \\
& r_{33}=-C_{5} S_{a b c} \\
& P_{x}=\left(L_{1} S_{3}+L_{2} S_{a b}\right) S_{1}-\left(L_{1} C_{3}+L_{2} C_{a b}\right) C_{1} S_{5} \\
& P_{y}=-\left(L_{1} S_{3}+L_{2} S_{a b}\right) C_{1}-\left(L_{1} C_{3}+L_{2} C_{a b}\right) S_{1} S_{5} \\
& P_{z}=-\left(L_{1} C_{3}+L_{2} C_{a b}\right) C_{5}
\end{aligned}
$$

The basic right-leg forward kinematic result is $\left[{ }_{6}^{0} T\right]$. To calculate $\left[{ }_{F}^{C} T\right]$, the pose of the rightleg end-effector frame $\{\mathrm{F}\}$ with respect to the AK Biped Robot model chest reference frame $\{\mathrm{C}\}$, the following transform equation is used.

$$
\begin{array}{cc}
{\left[{ }_{F}^{C} T\right]=\left[{ }_{0}^{C} T\right]\left[{ }_{6}^{0} T\left(\theta_{1}, \theta_{5}, \theta_{3}, \theta_{7}, \theta_{11}, \theta_{9}\right)\right]\left[{ }_{F}^{6} T\right]} \\
{\left[{ }_{0}^{C} T\right]=\left[\begin{array}{cccc}
0 & -1 & 0 & -L_{\mathrm{TX}} \\
0 & 0 & -1 & -L_{\mathrm{TY}} \\
1 & 0 & 0 & L_{\mathrm{TZ}} \\
0 & 0 & 0 & 1
\end{array}\right] \quad\left[{ }_{\mathrm{F}}^{6} T\right]=\left[\begin{array}{cccc}
1 & 0 & 0 & L_{\mathrm{F}} \\
0 & 1 & 0 & 0 \\
1 & 0 & 1 & 0 \\
0 & 0 & 0 & 1
\end{array}\right]}
\end{array}
$$

The overall transform equation above can be evaluated numerically. Constants $L_{T X}, L_{T Y}, L_{T Z}$, and $L_{F}$ were given earlier.

Note that $\left[{ }_{0}^{\mathrm{C}} \mathrm{T}\right]$ and $\left[{ }_{\mathrm{F}}^{6} \mathrm{~T}\right]$ are not evaluated by any row in the $\mathrm{D}-\mathrm{H}$ parameter table, since there is no variable associated with these fixed homogeneous transformation matrices based on constant lengths and orientation. Instead, they are determined by inspection, using the rotation matrix and position vector components of the basic homogeneous transformation matrix definition.

\section{Forward Kinematic Validation}

As an example for of 6-DOF right-leg Given $\left(\theta_{1}, \theta_{5}, \theta_{3}, \theta_{7}, \theta_{11}, \theta_{9}\right)=\left(-10^{\circ},-30^{\circ},-20^{\circ},-35^{\circ}\right.$,$\left.45^{\circ}, 60^{\circ}\right)$

$$
\left.{ }_{\mathrm{F}}^{\mathrm{C}} T\right]=\left[\begin{array}{cccc}
-0.35 & 0.90 & -0.26 & -135.2 \\
-0.36 & -0.38 & -0.85 & -256.1 \\
-0.87 & -0.20 & 0.45 & 58.5 \\
0 & 0 & 0 & 1
\end{array}\right]
$$


These forward kinematic results were validated by comparing the symbolic formulae derived in this paper with a numerical approach in each case.

\section{CONCLUSION}

In this paper was presented the kinematic modeling of AK bipedal robot with 12 DOF. For that the D-H method was used for calculation the position and rotation of end-effector of AK biped robot.

Also, the result was validated in my MATLAB by developing and applying the D-H method.

The result shows the effective of this method.

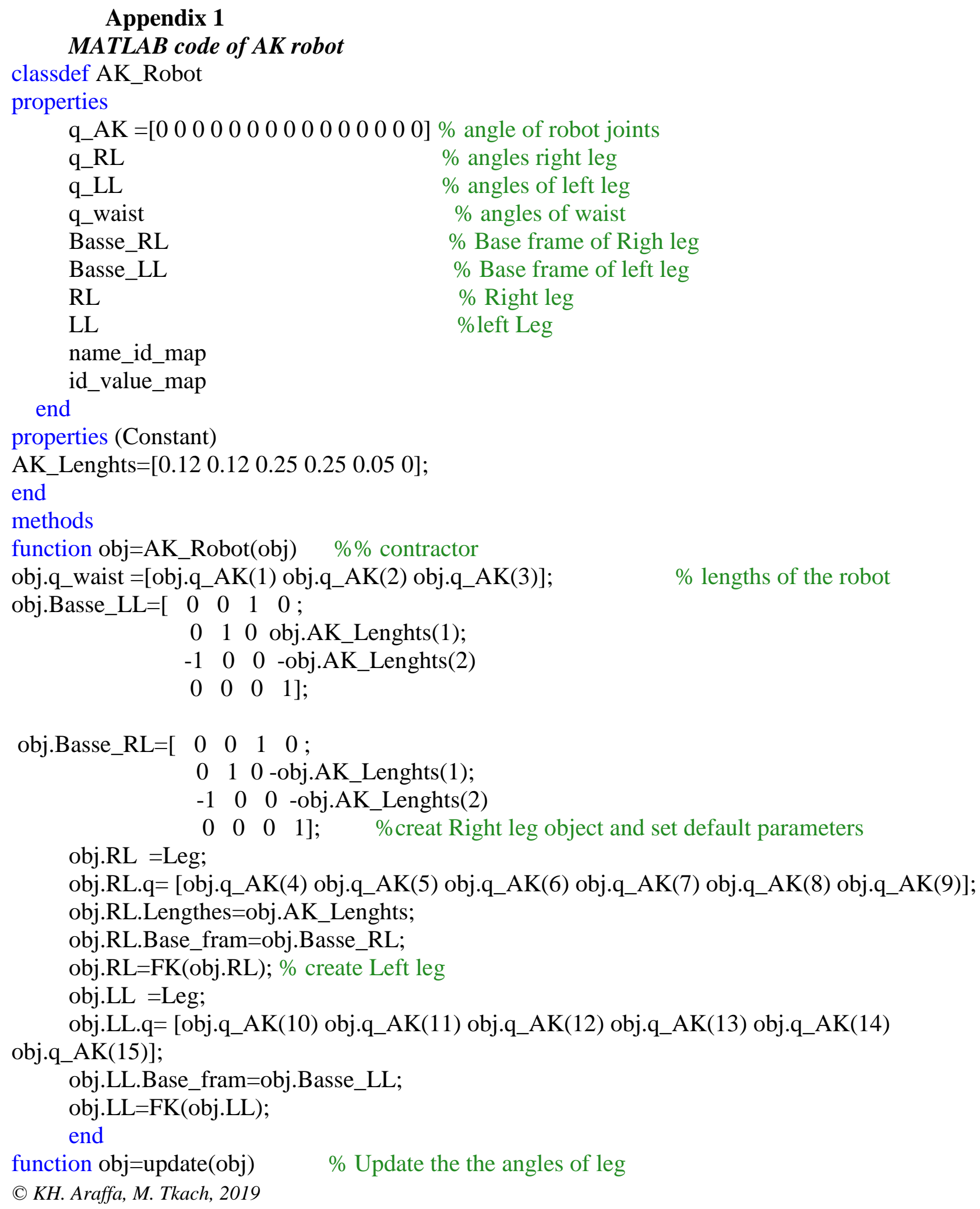


obj.RL.q= [obj.q_AK(4) obj.q_AK(5) obj.q_AK(6) obj.q_AK(7) obj.q_AK(8) obj.q_AK(9)];

obj.LL.q= [obj.q_AK(10) obj.q_AK(11) obj.q_AK(12) obj.q_AK(13) obj.q_AK(14)

end obj.q_AK(15)];

function obj=calc_FK(obj,angles)

obj.q_AK=angles;

obj=update $(o b j)$;

obj.RL=FK(obj.RL);

obj.LL=FK(obj.LL);

end

function obj=set_angles(obj,angles)

obj.q_AK=angles;

obj=obj.update () ;

end

end

end

\section{Appendix 2}

MATLAB class code of Leg and implementation D-H method for calculation forward classdef Leg \%AKA Robot Leg class (Khaldon araffa)

$\%$ Detailed explanation goes here

properties \%general

$\mathrm{AF}=\left[\begin{array}{llll}1 & 0 & 0 & 0.05\end{array}\right.$;

$\begin{array}{lllll}0 & 1 & 0 & 0 & \text {; }\end{array}$

$\begin{array}{lllll}0 & 0 & 1 & 0\end{array}$

$\left.\begin{array}{llllll}0 & 0 & 0 & 1\end{array}\right]$

Q; Lengthes;Base_fram

$\mathrm{X}$

$\%$ FK parametrs

$\mathrm{R}$

$\mathrm{P}$

A

$\mathrm{T}$

KNEE; ANKLE; HIP; ya; th11; th21; th31; th41; th51; th61; A1; A2; A3; A4; A5; theta; a end

properties (Constant)

$\mathrm{n}=6$;

offset $=\left[\begin{array}{llllll}0 & 0 & 0 & 0 & 0 & 0\end{array}\right]$;

type $=[$ 'r', 'r', 'r','r','r','r'];

$\mathrm{d}=\left[\begin{array}{llllll}0 & 0 & 0 & 0 & 0 & 0\end{array}\right]$;

base $=[0 ; 0 ; 0]$;

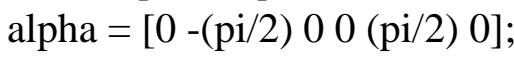

end

methods

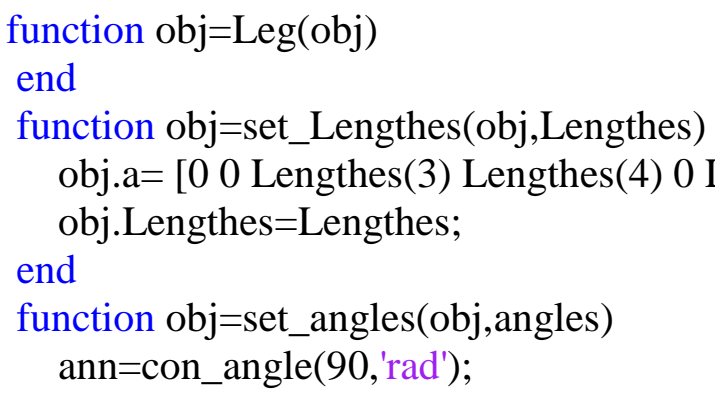




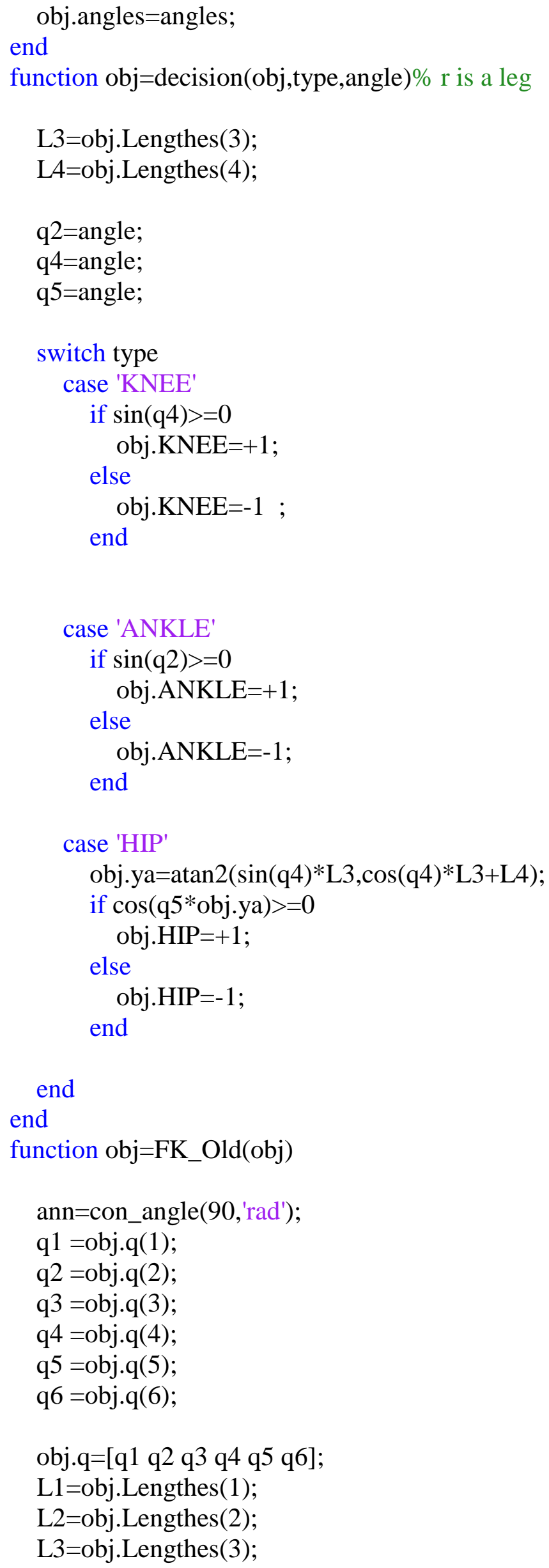


L4=obj.Lengthes(4);

L5=obj.Lengthes(5);

L6=obj.Lengthes(6);

obj.theta $=[\mathrm{q} 1+$ ann q2-ann q3+ann q4 q5 q6];

$\%$ dh parametts

alpha $=$ obj.alpha;

theta $=$ obj.theta;

$\mathrm{a}=$ obj.a;

$\mathrm{d}=$ obj.d;

base $=$ obj.base;

$\mathrm{Nx}=-\cos (\mathrm{q} 6) *(\sin (\mathrm{q} 1) * \sin (\mathrm{q} 3+\mathrm{q} 4+\mathrm{q} 5)-\cos (\mathrm{q} 1) * \cos (\mathrm{q} 2) * \cos (\mathrm{q} 3+\mathrm{q} 4+\mathrm{q} 5))-$

$\cos (\mathrm{q} 1) * \sin (\mathrm{q} 2) * \sin (\mathrm{q} 6)$;

$\mathrm{Ny}=\cos (\mathrm{q} 6) *(\cos (\mathrm{q} 1) * \sin (\mathrm{q} 3+\mathrm{q} 4+\mathrm{q} 5)+\cos (\mathrm{q} 2) * \cos (\mathrm{q} 3+\mathrm{q} 4+\mathrm{q} 5))-$

$\sin (\mathrm{q} 1) * \sin (\mathrm{q} 2) * \sin (\mathrm{q} 6)$

$\mathrm{Nz}=\cos (\mathrm{q} 2) * \sin (\mathrm{q} 6)+\cos (\mathrm{q} 3+\mathrm{q} 4+\mathrm{q} 5) * \cos (\mathrm{q} 6) * \sin (\mathrm{q} 2)$;

$\mathrm{Sx}=\sin (\mathrm{q} 6) *(\sin (\mathrm{q} 1) * \sin (\mathrm{q} 3+\mathrm{q} 4+\mathrm{q} 5)-\cos (\mathrm{q} 1) * \cos (\mathrm{q} 2) * \cos (\mathrm{q} 3+\mathrm{q} 4+\mathrm{q} 5))-$

$\cos (\mathrm{q} 1) * \cos (\mathrm{q} 6) * \sin (\mathrm{q} 2)$;

$\operatorname{Sy}=-\sin (\mathrm{q} 6) *(\cos (\mathrm{q} 1) * \sin (\mathrm{q} 3+\mathrm{q} 4+\mathrm{q} 5)+\cos (\mathrm{q} 2) * \cos (\mathrm{q} 3+\mathrm{q} 4+\mathrm{q} 5) * \sin (\mathrm{q} 1))-$

$\cos (\mathrm{q} 6) * \sin (\mathrm{q} 1) * \sin (\mathrm{q} 2)$;

$\mathrm{Sz}=\cos (\mathrm{q} 2) * \cos (\mathrm{q} 6)-\cos (\mathrm{q} 3+\mathrm{q} 4+\mathrm{q} 5)$;

$A x=\cos (q 3+q 4+q 5) * \sin (q 1)+\cos (q 1) * \cos (q 2) * \sin (q 3+q 4+q 5)$;

$A y=\cos (q 2) * \sin (q 1) * \sin (q 3+q 4+q 5)-\cos (q 1) * \cos (q 3+q 4+q 5)$;

$\mathrm{Az}=\sin (\mathrm{q} 2) * \sin (\mathrm{q} 3+\mathrm{q} 4+\mathrm{q} 5)$;

$\mathrm{Px}=\mathrm{L} 3 * \cos (\mathrm{q} 1) * \cos (\mathrm{q} 2) * \cos (\mathrm{q} 3)-$

$(\cos (\mathrm{q} 3) * \sin (\mathrm{q} 1)+\cos (\mathrm{q} 1) * \cos (\mathrm{q} 2) * \sin (\mathrm{q} 3)) *(\mathrm{~L} 4 * \sin (\mathrm{q} 4)+\mathrm{L} 5 * \cos (\mathrm{q} 6) * \sin (\mathrm{q} 4+\mathrm{q} 5)) \ldots$

$-(\sin (\mathrm{q} 1) * \sin (\mathrm{q} 3)-$

$\cos (\mathrm{q} 1) * \cos (\mathrm{q} 2) * \cos (\mathrm{q} 3)) *(\mathrm{~L} 4 * \cos (\mathrm{q} 4)+\mathrm{L} 5 * \cos (\mathrm{q} 6) * \cos (\mathrm{q} 4+\mathrm{q} 5))-$

$\mathrm{L} 3 * \sin (\mathrm{q} 1) * \sin (\mathrm{q} 3)+\mathrm{L} 5 * \cos (\mathrm{q} 1) * \sin (\mathrm{q} 2) * \sin (\mathrm{q} 6)$;

$\mathrm{Py}=(\cos (\mathrm{q} 1) * \sin (\mathrm{q} 3)+\cos (\mathrm{q} 2) * \cos (\mathrm{q} 3) * \sin (\mathrm{q} 1)) *(\mathrm{~L} 4 * \cos (\mathrm{q} 4)+\mathrm{L} 5 * \cos (\mathrm{q} 6) * \cos (\mathrm{q} 4+\mathrm{q} 5)) \ldots$ $+(\cos (\mathrm{q} 1) * \cos (\mathrm{q} 3)-$

$\cos (\mathrm{q} 2) * \sin (\mathrm{q} 1) * \sin (\mathrm{q} 3)) *(\mathrm{~L} 4 * \sin (\mathrm{q} 4)+\mathrm{L} 5 * \cos (\mathrm{q} 6) * \sin (\mathrm{q} 4+\mathrm{q} 5))+\mathrm{L} 3 * \cos (\mathrm{q} 1) * \sin (\mathrm{q} 3)+\mathrm{L} 3 * \cos (\mathrm{q} 2) *$ $\cos (\mathrm{q} 3) * \sin (\mathrm{q} 1)-\mathrm{L} 5 * \sin (\mathrm{q} 1) * \sin (\mathrm{q} 2) * \sin (\mathrm{q} 6)$;

$\mathrm{Pz}=\mathrm{L} 3 * \cos (\mathrm{q} 3) * \sin (\mathrm{q} 2)+\mathrm{L} 5 * \cos (\mathrm{q} 2) * \sin (\mathrm{q} 6)+\cos (\mathrm{q} 3) * \sin (\mathrm{q} 2) *(\mathrm{~L} 4 * \cos (\mathrm{q} 4)+\mathrm{L} 5 * \cos (\mathrm{q} 6) * \cos (\mathrm{q} 4+\mathrm{q}$ $5))-\sin (\mathrm{q} 2) * \sin (\mathrm{q} 3) *(\mathrm{~L} 4 * \sin (\mathrm{q} 4)+\mathrm{L} 5 * \cos (\mathrm{q} 6) * \sin (\mathrm{q} 4+\mathrm{q} 5))$;

$\mathrm{B}=[\mathrm{Nx}$ Sx Ax Px;

Ny Sy Ay Py;

$\mathrm{Nz} \mathrm{Sz} \mathrm{Az} \mathrm{Pz;}$

$\left.\begin{array}{llll}0 & 0 & 0 & 1]\end{array}\right]$

$\%$ return

obj.A=obj.Base_fram*B;

obj. $\mathrm{R}=\mathrm{B}(1: 3,1: 3)$;

obj. $\mathrm{P}=\mathrm{B}(1: 3,4)$;

end

function $\mathrm{obj}=\mathrm{FK}(\mathrm{obj})$

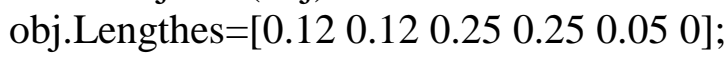

(C) KH. Araffa, M. Tkach, 2019 


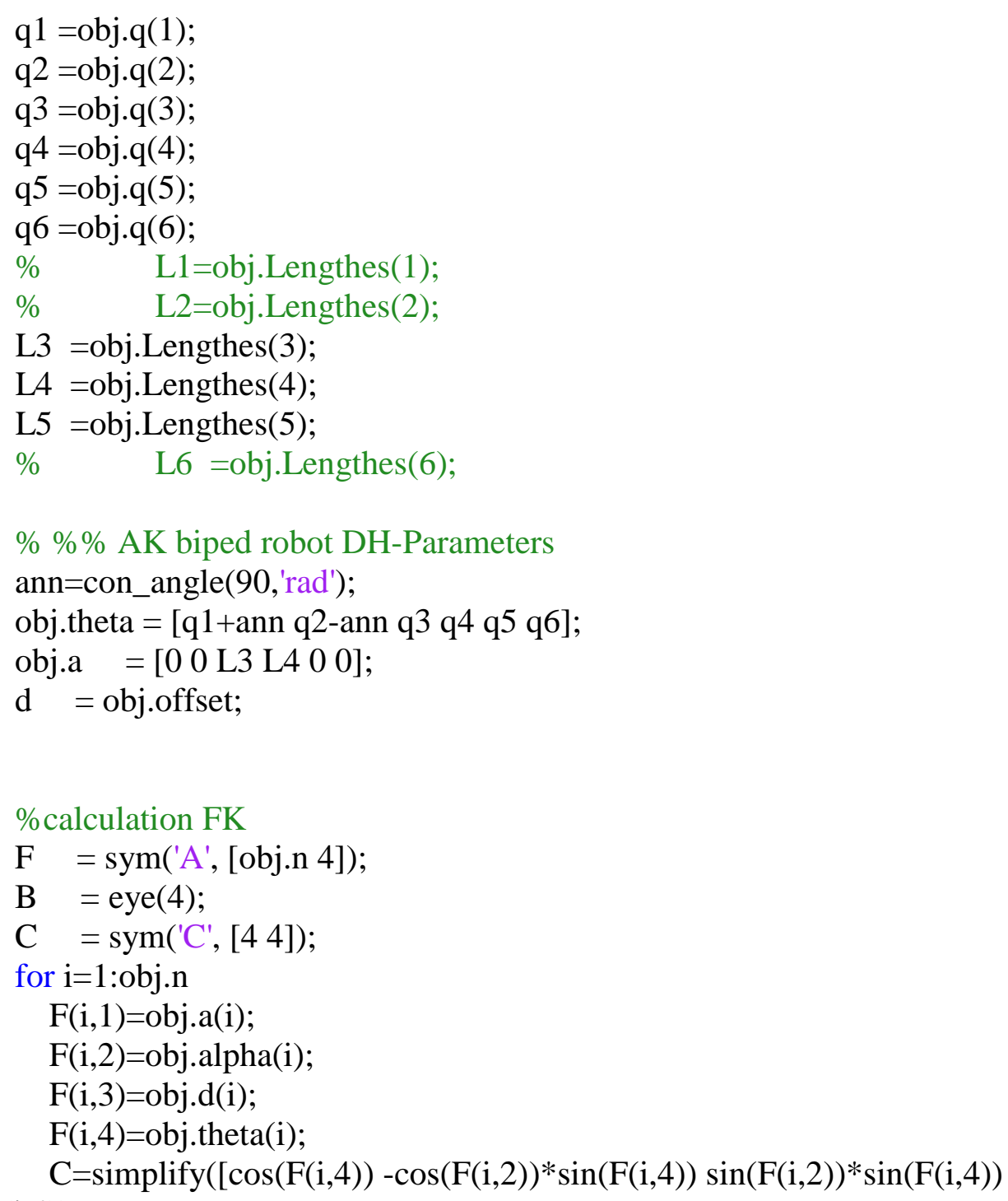

$\mathrm{F}(\mathrm{i}, 1) * \cos (\mathrm{F}(\mathrm{i}, 4))$;

$\sin (\mathrm{F}(\mathrm{i}, 4)) \cos (\mathrm{F}(\mathrm{i}, 4)) * \cos (\mathrm{F}(\mathrm{i}, 2))-\sin (\mathrm{F}(\mathrm{i}, 2)) * \cos (\mathrm{F}(\mathrm{i}, 4)) \mathrm{F}(\mathrm{i}, 1) * \sin (\mathrm{F}(\mathrm{i}, 4)) ;$

$0 \sin (\mathrm{F}(\mathrm{i}, 2)) \cos (\mathrm{F}(\mathrm{i}, 2)) \mathrm{F}(\mathrm{i}, 3)$;

$\left.\left.\begin{array}{llll}0 & 0 & 0 & 1\end{array}\right]\right)$;

genvarname('A', num2str(i));

eval(['A' num $\left.\left.2 \operatorname{str}(\mathrm{i}){ }^{\prime}=\mathrm{C}^{\prime}\right]\right)$

eval(sprintf('obj.A\%d = C;',i));

$\mathrm{B}=\mathrm{B} * \mathrm{C}$;

end

\%assign output

obj.A=double(obj.Base_fram*B*obj.AF);

obj.R=obj.A(1:3,1:3); \% R rotation matrix

obj.P=obj.A(1:3,4); \%'P is translation Matrix'

end

end

end 


\section{REFERENCES}

1. P. Corke, "Denavit-Hartenberg notation for common robots," no. March, pp. 1-14, 2014.

2. C. Hernández-Santos, E. Rodriguez-Leal, R. Soto, and J. L. Gordillo, "Kinematics and dynamics of a new 16 DOF humanoid biped robot with active toe joint," Int. J. Adv. Robot. Syst., 2012, DOI: 10.5772/52452.

3. X. Zhao and Y. Liu, "Modeling of biped robot," 2010 Chinese Control Decis. Conf. CCDC 2010, pp. 3233-3238, 2010, DOI: 10.1109/CCDC.2010.5498610.

4. S. Kucuk and Z. Bingul, Robot Kinematics - Forward and Inverse Kinematics, no. December. 2007, ISBN: 3-86611-285-8.

5. J. J. Craig, Introduction to Robotics, 3rd ed., vol. 1, no. 2. 1986, ISBN: 0-13-123629-6. 